\title{
PASS COMPLETION RATE AND MATCH OUTCOME AT THE WORLD CUP IN BRAZIL IN 2014
}

\author{
MICHAŁ KRÓL1, MAREK KONEFAŁ¹, PAWEŁ CHMURA², MARCIN ANDRZEJEWSKI³, \\ TOMASZ ZAJAC ${ }^{4}$, JAN CHMURA ${ }^{1}$ \\ IUniversity School of Physical Education in Wroclaw, Faculty of Sport Sciences, \\ Chair of Biological and Motor Aspects of Sports \\ 2University School of Physical Education in Wroclaw, Faculty of Physical Education, Chair of Team Games \\ ${ }^{3}$ Poznan University of Physical Education, Faculty of Tourism and Recreation, \\ Department of Recreation \\ ${ }^{4}$ Jerzy Kukuczka Academy of Physical Education in Katowice, Faculty of Physical Education, \\ Function Testing Laboratory
}

\author{
Mailing address: Paweł Chmura, University School of Physical Education in Wroclaw, Chair of Team Games, \\ 35 I.J. Paderewskiego Avenue, 51-612 Wrocław, tel.: +48 71 3473564, fax: +48 71 3473562, \\ e-mail: pawel.chmura@awf.wroc.pl
}

\begin{abstract}
Introduction. Having possession of the ball makes it possible for the players of a team to undertake a greater number of actions that can help the team win the match. The aim of the current study was to determine the number and completion rate of passes made from different distances in relation to percentage ball possession and the outcome of the match in the group stage of the FIFA World Cup held in Brazil in 2014. Material and methods. The study included 32 teams who participated in the final tournament. The performance of 266 players (682 observations) who played 48 matches in the group stage was examined. Only players who took part in the entire match were included in the research, and goal-keepers were excluded. The data used in the study, which had been recorded by means of the Castrol Performance Index system, were obtained from the FIFA official website. The total number of passes and the percentage of completed passes were analysed, which were classified as short, medium, and long, in relation to the team's percentage possession of the ball (over and below 50\%) and the outcome of the match (win, loss, or draw). Results. Out of the three types of passes which were examined in the study, teams playing in the World Cup in Brazil most often made medium passes. The number of these passes was the highest in matches won. These matches were also characterised by a significantly higher percentage of completed long passes. Conclusions. Players from teams who were in possession of the ball more than $50 \%$ of the time performed a higher number of all types of passes, regardless of the outcome of the match. The completion rate for these players was significantly higher only for short and medium passes.
\end{abstract}

Key words: passes, completion rate, ball possession, match outcome, soccer

\section{Introduction}

In modern soccer, there is a tendency for the game to be played at an increasingly rapid pace. In this situation, many teams decide to adopt a strategy of short passes, which makes it possible to minimise the risk of losing the ball and, at the same time, increases percentage ball possession during the game [1, 2]. Players who take part in the final tournament of the World Cup need to be adequately prepared physically, mentally, as well as technically and tactically [3]. Matches are held every few days, and each team plays seven matches in a month, which means that the players play for a total of 630 minutes, not including extra time that may be added in the knockout stage. The best players cover a distance of approximately $84 \mathrm{~km}$ during the final tournament, which is the equivalent of two marathon runs [4]. The players' match activity profiles are varied and consist of walking; running with low, moderate, and high speed; as well as sprinting, running backwards, and running sideways. Players thus make use of various energy resources that are produced in aerobic processes (low-intensity running) as well as in anaerobic processes (high-intensity running and sprinting), and these bouts of more intense effort are interspersed with periods of standing and walking, which, when combined, account for more than half of the time of match play $[5,6]$.

The actions performed in soccer can be categorised according to whether or not they involve possession of the ball and whether they are offensive or defensive [7]. One of the important elements of team collaboration is passing the ball. Buraczewski [8] found that during seven consecutive final tournaments of the World Cup (from 1978 to 2002), the technique and completion rate of passes as well the number of passes in various parts of the field changed; the passes which were made the most often were moderate passes from a distance of 10 to 29.9 metres. According to this author, when teams with similar skill levels play against each other, the number of passes made does not correlate with the outcome of the match. However, another author has observed a tendency for higher-ranked teams to make more passes during the match than those with lower skill levels when two teams with different skill levels play against each other [9].

The research carried out by Lago et al. [10] in the years 2003 and 2004 based on 170 matches of the Spanish Primera Division showed that ball possession and the location where the match was played determined the outcome of the match. Ball possession was greater in teams who won the match and those 
who played at home. The amount of time a team possesses the ball has also been found to depend on the ranking of the team and the abilities of individual players [7]. Having possession of the ball makes it possible to undertake many types of actions that can help the team win the match. Bradley et al. [1], who analysed the performance of teams playing in the English Premier League, found that players whose teams were characterised by high percentage ball possession made $44 \%$ more passes than their opponents did. This trend was also observed for the effectiveness of passes, goal attempts, ball reception, and dribbling.

Performance outcomes in soccer depend on several factors, including the players' skills [11], their motor preparation [12], and the tactics [13]. The outcome may also be influenced by external factors, such as whether the game is played at home or away, the temperature and humidity [14], the altitude [15], and the quality of the opponent [16]. Many coaches emphasise the fact that success in football matches is also dependent upon the players' volitional features and motivation [17], as well as their mental predispositions and the efficiency of their analytical and decision-making processes [18].

Hoppe et al. [19], who performed an analysis of 306 Bundesliga matches in the 2012/2013 season, found that in soccer, success is achieved, among others, thanks to the abilities of individual players combined with the team's possession of the ball. From a cognitive and practical point of view, it would be interesting to determine whether such relationships can be observed during the most prestigious soccer tournament. In modern soccer, the players' skills are highly important, and these include in particular the ability to make passes from different distances and perform them successfully [1]. However, scarce research is available concerning how the distance from which passes are made and ball possession are related to the outcomes achieved by the highest-ranked players. The aim of the current study was to determine the number and completion rate of passes made from different distances and to investigate their relationship with percentage ball possession and the outcome of the match in the group stage of the World Cup in soccer held in Brazil in 2014.

\section{Material and methods}

\section{Data set}

The study included 32 teams who participated in the final tournament of the World Cup in Brazil in 2014. The performance of 266 players (682 observations) who played 48 matches in the group stage was analysed. Only players who took part in the entire match were included in the research, and goal-keepers were excluded. The data, which had been recorded using the
Castrol Performance Index system, were obtained from the FIFA official website, where they had been made accessible in the form of reports (www.fifa.com/worldcup/archive/brazil2014/ index.html).

\section{Procedure}

The Castrol Performance Index is a kinematic analysis system which performs analyses in real time based on data recorded using semi-automatic cameras at 25 frames per second. The system, which was first introduced in 2008, makes it possible to analyse the game in an objective way. It analyses approximately 1,800 movements made by each player per match, evaluating whether their actions assisted or prevented goals. The process of data collection is complex; it is possible to track player movement in each second of the game and in all parts of the field at the same time. Each of the movements of the players is described using quantitative values. As a result, information is obtained as to the players' activity during the match, including its intensity, volume, and form, as well as some of the skills of the players $[20,21,22]$. In the current study, the total number of passes and the percentage of completed passes were analysed, which were classified as short (0.1-9.99 m), medium (10-29.99 m) and long (over $29.99 \mathrm{~m}$ ), in relation to the team's percentage possession of the ball (over and below 50\%) and the outcome of the match (win, loss, or draw).

\section{Statistical analysis}

The data were subjected to statistical analysis, which was performed using Statistica 12.0. Normality of distribution was tested using the Shapiro-Wilk test. Mean values and standard deviations were calculated. Multivariate analysis of variance was used, the two factors being match outcome and percentage ball possession. The significance of the differences between means was determined using Fisher's LSD test. Statistical significance was set at $\mathrm{p} \leq 0.05$.

\section{Results}

In the analysis of the number of passes made by the players during the group stage of the World Cup in Brazil, a statistically significantly higher number of medium passes was found in matches that ended in a win or draw compared to matches lost, but only if the team possessed the ball more than $50 \%$ of the time during the game $(\mathrm{p}<0.05)$. As for the total number of passes, significant differences were also noted between matches won and lost when the team had more than $50 \%$ of ball possession $(\mathrm{p}<0.05)$ (tab. 1).

Table 1. Number of passes made in relation to percentage ball possession and match outcome

\begin{tabular}{|c|c|c|c|c|c|c|}
\hline Ball possession & \multicolumn{3}{|c|}{ Over $50 \%$} & \multicolumn{3}{|c|}{ Below $50 \%$} \\
\hline Match outcome & Win & Draw & Loss & Win & Draw & Loss \\
\hline Pass number & \multicolumn{6}{|c|}{$\bar{x} \pm S D$} \\
\hline Short & $14.78 \pm 7.15$ & $13.64 \pm 5.30$ & $13.74 \pm 6.63$ & $9.70 \pm 4.98$ & $9.52 \pm 5.42$ & $10.14 \pm 5.03$ \\
\hline Medium & $38.22 \pm 16.60^{*}$ & $38.48 \pm 15.10 \#$ & $34.37 \pm 13.77^{*} \#$ & $22.34 \pm 7.66$ & $25.50 \pm 11.51$ & $24.89 \pm 10.50$ \\
\hline Long & $7.79 \pm 5.09$ & $7.34 \pm 5.03$ & $7.33 \pm 4.82$ & $5.28 \pm 3.17$ & $6.16 \pm 3.43$ & $5.45 \pm 3.80$ \\
\hline All & $60.79 \pm 22.48^{*}$ & $59.47 \pm 19.77$ & $55.43 \pm 18.29^{*}$ & $37.33 \pm 11.7$ & $41.17 \pm 16.26$ & $40.47 \pm 14.33$ \\
\hline
\end{tabular}

* = statistically significant differences between win and loss $(\mathrm{p}<0.05)$.

$\#$ = statistically significant differences between draw and loss $(\mathrm{p}<0.05)$. 
Table 2. Pass completion rate in relation to percentage ball possession and match outcome

\begin{tabular}{|c|c|c|c|c|c|c|}
\hline Ball possession & \multicolumn{3}{|c|}{ Over $50 \%$} & \multicolumn{3}{|c|}{ Below $50 \%$} \\
\hline Match outcome & Win & Draw & Loss & Win & Draw & Loss \\
\hline $\begin{array}{l}\text { Pass completion } \\
\text { rate [\%] }\end{array}$ & \multicolumn{6}{|c|}{$\bar{x} \pm S D$} \\
\hline Short & $82.20 \pm 12.77$ & $79.56 \pm 14.69$ & $80.86 \pm 12.96$ & $75.22 \pm 18.12$ & $72.69 \pm 20.70$ & $75.15 \pm 19.25$ \\
\hline Medium & $83.82 \pm 10.49$ & $80.59 \pm 12.70$ & $80.86 \pm 12.60$ & $75.79 \pm 11.33$ & $75.84 \pm 12.20$ & $76.71 \pm 14.05$ \\
\hline Long & $62.35 \pm 28.08^{*} x$ & $51.42 \pm 28 x$ & $55.60 \pm 28.00^{*}$ & $56.32 \pm 29.97$ & $51.34 \pm 28.65$ & $53.21 \pm 29.85$ \\
\hline All & $80.78 \pm 9.53^{\star} x$ & $77.50 \pm 10.83 x$ & $78.17 \pm 10.53^{*}$ & $72.88 \pm 10.00$ & $72.26 \pm 10.83$ & $73.72 \pm 12.10$ \\
\hline
\end{tabular}

* = statistically significant differences between win and loss $(\mathrm{p}<0.05)$.

$\mathrm{x}=$ statistically significant differences between win and draw $(\mathrm{p}<0.05)$.

The analysis of the percentage pass completion rate revealed that in matches that were won players were characterised by a significantly greater percentage of completed long passes compared to matches that ended in a draw or loss $(p<0.05)$ if ball possession was over $50 \%$. Moreover, the completion rate of all of the passes was significantly higher in matches won than in those drawn and lost (tab. 2).

Teams who were in possession of the ball more than $50 \%$ of the time during the game performed a significantly higher total number of all types of passes and had a significantly higher pass completion rate for short and medium passes in matches won, drawn, and lost compared to teams who were in control of the ball less than $50 \%$ of the time ( $<<0.05)$. No statistically significant differences were found for the completion rate of long passes between teams with over and below $50 \%$ of ball possession in any matches regardless of the outcome (tab. 1, 2).

\section{Discussion}

Modern soccer requires that players perform a considerable amount of different types of locomotor activity. This includes motor actions and actions with the ball that are based on the individual abilities of particular players. The actions which are performed the most often by the players are passes, whose effectiveness often determines the outcome of the match [1, 16, 23].

The current study found that during the group stage of the World Cup in Brazil, the players whose teams were in possession of the ball more than $50 \%$ of the time during the match were characterised by a significantly higher number of medium passes in matches won and drawn than in matches lost. Barnes et al. [24], who compared seasons 2006/2007 and 2012/2013 in the Premier League, observed that in the latter season, the players made a significantly higher number of passes $(25.3 \pm$ 13.4 vs. $35.4 \pm 17.1$ ). They also found that the players performed the greatest number of medium passes $(19.8 \pm 11.3)$, similarly as in our study, where, regardless of the outcome of the match, the players performed from 22.3 up to as many as 38.2 medium passes. According to Michailidis et al. [25], during the UEFA European Championship in Poland and the Ukraine in 2012, the greatest number of goals (18.4\%) were scored from short passes (up to $10 \mathrm{~m}$ ), and $17.1 \%$ were scored from medium and long passes (more than $10 \mathrm{~m}$ ). When the same authors [25] analysed the matches of the European Champions League, they found that short passes were the most prominent type of action undertaken by the players which led to scoring a goal (25.0\%), whereas medium and long passes contributed to scoring only $8.4 \%$ of all goals. The differences between the findings of Michailidis et al.'s study and ours are due to the fact that our analyses included the passes made during the entire match, whereas theirs only included those performed immediately before goals were scored. The outcome of the match is determined not only by situations which occur immediately before the goal is scored, though they are the most important, but also, crucially, on several other actions undertaken during the entire game [26].

Our study revealed that teams who were in possession of the ball over $50 \%$ of the time and won the match had a completion rate of $83.82 \%$ for medium passes, and this rate was the highest of the rates for all the passes. In modern soccer, using medium passes is the most effective way to move the ball forward towards the opponent's goal. On the one hand, medium passes are a faster way to move the ball than short passes, and on the other, they have a better completion rate than long passes [27]. Despite the higher effectiveness of medium passes, we observed that in matches won, there was a higher percentage completion rate of long passes than in matches drawn and lost. This could have been caused, among others, by the superior skills of individual players, who were able to use long passes in order to gain a numerical advantage in parts of the field located immediately next to the opponent's goal. Barnes et al. [24] found that in seven consecutive seasons of the Premier League, the completion rate of the passes made by the players increased from $76.3 \%$ to $83.3 \%$. This information, combined with that concerning the rise in the number of short and medium passes (with minor changes in the number of long passes), suggests that there was an increase in the number of passes in the last few seasons, as a result of the game becoming more intense [28, 29].

The outcome of the game is affected by percentage ball possession and the number of passes made by the team. The research by Lago-Peñas et al. [27] demonstrated that in elite league tournaments (the French, German, Spanish, and English leagues), ball possession did not have an impact on the result of the match in teams whose players had superior individual abilities. However, in teams with players whose skill levels are lower, ball possession tended to decrease from the moment when the team achieved a favourable outcome. This demonstrates that the best teams strive to have possession of the ball for as long a time as possible regardless of the outcome of the match [23]. On the other hand, teams that have players with lower skill levels tend to adopt a defensive strategy in order to maintain the favourable outcome of the match, which causes their percentage ball possession to decrease [2, 30, 31]. Our study has shown that players from teams who were in possession of the ball more 
than $50 \%$ of the time made a significantly greater number of all types of passes; regardless of the distance from which the passes were made, they performed $60.79 \pm 22.48$ passes in matches won, $59.47 \pm 19.77$ passes in matches drawn, and $55.43 \pm 18.29$ in matches lost. Performing a large number of passes which are completed successfully determines maintaining possession of the ball. When analysing the completion rate of the passes made during the World Cup in Brazil, we found that only long passes were not associated with percentage ball possession. This finding was probably due to the small number of long passes both for situations when the teams had more and less than $50 \%$ of ball possession [23]. The issue of determining the association between the number and completion rate of passes and the outcome of the match is important, and further research needs to be performed in this respect.

\section{Conclusions}

Teams playing in the group stage of the World Cup in Brazil most often made medium passes. These passes were performed significantly more frequently in matches won and drawn. Moreover, the matches that ended in a win were characterised by a significantly higher number of medium passes and a significantly higher percentage completion rate for long passes. That is why the training process should include practising performing a high number of such passes.

Irrespective of the outcome of the match, teams who were in possession of the ball over $50 \%$ of the time performed a greater number of short, medium, and long passes. Only in the case of long passes was there no association between percentage ball possession and pass completion rate.

\section{Literature}

1. Bradley P.S., Lago-Peñas C., Rey E., Gomez Diaz A. (2013). The effect of high and low percentage ball possession on physical and technical profiles in English FA Premier League soccer matches. Journal of Sports Sciences 31(12), 126170 .

2. Collet C. (2013). The possession game? A comparative analysis of ball retention and team success in European and international football, 2007-2010. Journal of Sports Sciences 31(2), 123-36.

3. Drust B., Ahmed Q., Roky R. (2012). Circadian variation and soccer performance: Implications for training and matchplay during Ramadan. Journal of Sports Sciences 30(Suppl 1), S43-S52.

4. Chmura P., Goliński D., Andrzejewski M., Mroczek D., Kawczyński A., Rokita A. et al. (2014). Endurance capacity and selected technical-tactical skills of soccer players participating in the group stage of the 2014 FIFA World Cup. Trends in Sport Sciences 3(21), 153-160.

5. Bangsbo J. (1994). Energy demands in competitive soccer. Journal of Sports Sciences 12(Special Issue), S5-S12.

6. Stolen T., Chamari K., Castagna C., Wisloff U. (2005). Physiology of soccer: An update. Sports Medicine 35, 501-536.

7. Vogelbein M., Nopp S., Hökelmann A. (2014). Defensive transition in soccer - are prompt possession regains a measure of success? A quantitative analysis of German Fußbal1-Bundesliga 2010/2011. Journal of Sports Sciences 32(11), 1076-83.

8. Buraczewski T. (2004). Pass technique and quality tendencies in high rank football matches. In J. Bergier (ed.), $\mathrm{Ob}$ - servation and assessment of players' performance in team sport games. Wrocław: MTNG. [in Polish]

9. Soroka A. (2011). Characteristics of selected models of footballers' activity during the World Cup - South Africa 2010. Biała Podlaska: PSW. [in Polish]

10. Lago C., Martin R. (2007). Determinants of possession of the ball in soccer. Journal of Sports Sciences 25(9), 969-74.

11. Rampinini E., Impellizzeri F.M., Castagna C., Coutts A.J., Wisløff U. (2009). Technical performance during soccer matches of the Italian Serie A league: Effect of fatigue and competitive level. Journal of Sports Science and Medicine 12(1), 227-33.

12. Chmura J., Dargiewicz R., Andrzejewski M. (2004). The endurance and speed abilities of players during the qualifying game to the Champions League in football. In J. Bergier (ed.), Observation and assessment of players' performance in team sport games. Wrocław: MTNG. [in Polish]

13. Tenga A., Holme I., Ronglan L.T., Bahr R. (2010). Effect of playing tactics on goal scoring in Norwegian professional soccer. Journal of Sports Sciences 28(3), 237-44.

14. Konefał M., Chmura P., Andrzejewski M., Chmura J. (2014). Analysis of motor performance of professional soccer players in different environmental conditions. Trends in Sport Sciences 4(21), 221-227.

15. Wehrlin J.P., Hallén J. (2006). Linear decrease in VO2max and performance with increasing altitude in endurance athletes. European Journal of Applied Physiology 96(4), 404-12.

16. Liu H., Hopkins W.G., Gómez M.A. (2016) Modelling relationships between match events and match outcome in elite football. European Journal of Sport Science 16(5), 516-25.

17. Payne S.M., Hudson J., Akehurst S., Ntoumanis N. (2013). Development and initial validation of the Impression Motivation in Sport Questionnaire - Team. International Journal of Sport and Exercise Psychology 35(3), 281-98.

18. Lex H., Essig K., Knoblauch A., Schack T. (2015). Cognitive representations and cognitive processing of team-specific tactics in soccer. PLoS One 10(2), e0118219.

19. Hoppe M.W., Slomka M., Baumgart C., Weber H., Freiwald J. (2015). Match running performance and success across a season in German Bundesliga soccer teams. International Journal of Sports Medicine 36(7), 563-6.

20. Duk O.S., Min K.S., Kawczyński A., Chmura P., Mroczek D., Chmura J. (2010). Endurance and speed capacity of the Korea Republic football national team during the World Cup of 2010. Journal of Human Kinetics 30, 115-21.

21. Soroka A. (2014). The locomotor activity of football players based on playing positions during the 2010 World Cup. The Journal of Sports Medicine and Physical Fitness Epub Ahead of print.

22. Nassis G.P., Brito J., Dvorak J., Chalabi H., Racinais S. (2015). The association of environmental heat stress with performance: Analysis of the 2014 FIFA World Cup Brazil. British Journal of Sports Medicine 49, 609-13.

23. da Mota G.R., Thiengo C.R., Gimenes S.V., Bradley P.S. (2016). The effects of ball possession status on physical and technical indicators during the 2014 FIFA World Cup Finals. Journal of Sports Sciences 34(6), 493-500.

24. Barnes C., Archer D.T., Hogg B., Bush M., Bradley P.S. (2014). The evolution of physical and technical performance parameters in the English Premier League. International Journal of Sports Medicine 35(13), 1095-100.

25. Michailidis Y., Michailidis C., Primpa E. (2013). Analysis of goals scored in European Championship 2012. Journal of Human Sport and Exercise 8(2), 367-375. 
26. Castellano J., Casamichana D., Lago C. (2012). The use of match statistics that discriminate between successful and unsuccessful soccer teams. Journal of Human Kinetics 31, 139-47.

27. Lago-Peñas C., Gómez-López M. (2014). How important is it to score a goal? The influence of the scoreline on match performance in elite soccer. Perceptual and Motor Skills 119(3), 774-84.

28. Bradley P.S., Sheldon W., Wooster B., Olsen P., Boanas P., Krustrup P. (2009) High-intensity running in English FA Premier League soccer matches. Journal of Sports Sciences 27, 159-168.

29. Bradley P.S, Carling C., Archer D., Roberts J., Dodds A., Di Mascio M. et al. (2011). The effect of playing formation on high-intensity running and technical profiles in English FA Premier League soccer matches. Journal of Sports Sciences 29(8), 821-30.

30. Di Salvo V., Gregson W., Atkinson G., Tordoff P., Drust B. (2009). Analysis of high intensity activity in Premier League soccer. International Journal of Sports Medicine 30(3), 205-12.

31. Lago C. (2009). The influence of match location, quality of opposition, and match status on possession strategies in professional association football. Journal of Sports Sciences 27(13), 1463-9.

Submitted: July 25, 2016

Accepted: December 1, 2016 